\title{
What matters for adolescent suicidality: depressive symptoms or fixed mindsets? Examination of cross-sectional and longitudinal associations between fixed mindset and suicidal ideation
}

\author{
SHIMIN ZHU ${ }^{\mathrm{a}^{*}}$ AND PAUL WONG \\ a. Department of Applied Social Sciences, The Hong Kong Polytechnic \\ University \\ b. Department of Social Work and Social Administration, The University of \\ Hong Kong
}

\begin{abstract}
*Corresponding author:
Dr. Shimin ZHU

Department of Applied Social Sciences, Department of Applied Social Sciences, The Hong Kong Polytechnic University, Hung Hom, Kowloon, Hong Kong SAR, China

The address of the institution at which the work was carried out:

Department of Applied Social Sciences, The Hong Kong Polytechnic University, Kowloon, Hong Kong

Acknowledgments: This work was supported by the Hong Kong Research Grant Council (Ref: 25605418) awarded to Shimin Zhu. We would like to thank the participants of the study, and the schools and research assistants for their assistance with the participant recruitment and data collection.
\end{abstract}

Declaration of interest: None

Email: jasmine.zhu@polyu.edu.hk 


\begin{abstract}
Background: Fixed mindsets, or beliefs about the non-malleability of self-attributes have been linked to a wide range of clinical psychological outcomes. Yet, their impact on suicidality has not been examined.

Objectives: To examine the association of fixed mindset of depression, anxiety and stress and suicidal ideation (SI) and its mediating role underlying the association between depression and SI.

Methods: A sample of 1393 adolescents $\left(\mathrm{M}_{\mathrm{age}}=13.04, \mathrm{SD}=0.85,640\right.$ boys $)$ from eleven middle schools voluntarily participated in a two-wave longitudinal study with 9-month interval.

Results: Both depression and fixed mindset positively and significantly associated with concurrent and future suicidality, after controlling demographic and socioeconomic status and previous SI. Participants with stronger fixed mindset presented stronger association with suicidality than those with only depressive symptoms. Also, fixed mindset of depression, anxiety and stress mediated the association between depression and suicidality in both cross-sectional and longitudinal models.

Conclusion: Findings of the current study not only shed light on the role of fixed mindset on suicidality and the mechanisms linking depression and suicidality among adolescents, but also provide an empirical basis for formulating prevention and/or intervention programs aimed at reducing the development of suicidality and minimizing the negative psychological reactions to challenges during human development.
\end{abstract}

Keywords: implicit theories, negative emotion, mediation model, middle school students, suicidal ideation 


\section{What matters for adolescent suicidality: depressive symptoms or fixed mindsets? Examination of cross-sectional and longitudinal associations between fixed mindset and suicidal ideation}

\section{INTRODUCTION}

Suicide prevention among young people is a global priority (World Health Organization, 2014). Identifying factors and understanding the mechanisms for the development of suicidality are main suicide prevention efforts. Risk factors for suicidality among young people have been identified that include inequitable social and demographic factors; personality and interpersonal factors; and mental well-being issues such as depression, anxiety, and substance abuse etc. (Chu et al., 2017; Dykxhoorn et al., 2017). Since not all identified risk factors are easily modifiable, examination of modifiable factors that predispose individuals to suicidality provides potential ways for saving young people lives.

Emerging literature has begun to examine the impact of implicit theories or mindsets belief of the malleability of personal attributes on mental well-being (e.g.,King \& dela Rosa, 2019; Kneeland, Dovidio, et al., 2016; Schroder, Kneeland, et al., 2019). According to the implicit theories, individuals with fixed mindsets believe their attributes are neither changeable nor improved by attempts (Dweck, 2013). For instance, those who believe emotions are fixed are associated with less active emotion regulation and are less engaged in regulatory efforts (Kneeland, Dovidio, et al., 2016); fixed mindsets of anxiety predicted future depressive symptom and negative affects (Schroder, Callahan, et al., 2019). On the other hand, believing in the flexibility of emotion is associated with more cognitive reappraisal of emotion regulation and higher subjective wellbeing among adolescents in adverse situations ( $\mathrm{Zhu}, \mathrm{Ni}$, et al., 2020). Young people with fixed mindsets were found to be more likely to have mental health problems (see a review, Schleider et al., 2015)). Fixed mindset of anxiety, in particular, was associated with less resilience, less effortful treatment, such as counselling other than medication, and more psychiatric symptoms (Schleider \& Schroder, 2018; Schroder, 2018; Schroder, Kneeland, et al., 2019).

It is noteworthy that the associations between mindsets and mental health may vary in different domains because mindsets are both domain-specific and domain-general (Schroder et al., 2016; Zhu, Zhuang, et al., 2020). Zhu and colleagues (2020) found that adolescents were more likely to think trait-related attributes (e.g., intelligence and personality) are fixed, while perceived state-related domains (e.g., emotion, feeling, and behavior) are more likely to change. It was also found that fixed mindset of depression predicted depression symptoms and fixed mindsets of anxiety were more strongly associated with hopelessness and mental health symptoms than the mindset of general emotions (Mullarkey \& Schleider, 2020; Schroder et al., 2016; Schroder, Kneeland, et al., 2019). 


\section{Depression and suicidality among adolescents}

Suicide is the fourth leading cause of death among 15-29 year-olds around the world (World Health Organization, 2021) and the presence of psychiatric disorders, especially depression, is known as one of the most robust contributing factors to the development of suicidality among young people (Evans et al., 2004; Olié et al., 2010). People with suicidal ideation are usually overwhelmed by painful emotions known as 'psych-ache' and see death as the 'only' solution for resolving some temporary and transitory life challenges (Olié et al., 2010). It is noteworthy that most people with psychiatric issues do not contemplate, attempt, nor complete suicide. In other words, if individuals may consider their negative emotions as temporary and malleable, they are more likely to feel less hopeless and be more resilient than those who consider sufferings are chronic and non-malleable (Kneeland, Dovidio, et al., 2016).

The association between mindsets and suicidality has not received much attention but it seems that fixed mindsets seem to play an important role in the suicidal process (Olié et al., 2010). Previous studies have found that mindsets differentially predict willingness to tolerate discomfort (Schroder, 2020), and having a fixed mindset may decrease hope for improvement and lead to avoidance coping and hopelessness. Suicidal thoughts emerge among distressed young people if they take a constricted mindset and think of death as the only way to escape the temporarily psych-ache (Tezanos et al., 2021). Examining the specific psychological process contributing to suicidal risk may leverage the suicide prevention or early intervention to better result. Changing fixed mindsets may be a potential prevention for suicide. A previous study of university students found that those with information about malleability of emotion engaged in more spontaneous cognitive reappraisal than did their counterparts who learned that emotions were fixed during an anxiety-provoking public speaking task (Kneeland, Holen-Hoeksema, et al., 2016). Similarly, compared to the manipulation to promote the growth mindset of depression among depressive individuals, the manipulation of the belief that depression is a chronic condition resulted in higher depression symptom severity and prognostic pessimism (Zimmermann et al., 2020). In addition, intervention studies teaching adolescents growth mindsets of personality in single-session interventions reported immediate increase in perceived control and longterm reduced anxiety and depression (Schleider, Abel, et al., 2019; Schleider, Burnette, et al., 2019). Fixed mindsets of depression, anxiety, and stress may also be a potential risk for suicide. Taking psychological sufferings as unchanged or incurable problems may be a more direct cause of a sense of hopeless and cue the idea that suicide is a possible way out of the pain (Shneidman, 1998).

This current study aims to examine the interaction mechanism among depression, fixed mindset of depression, anxiety, and stress and suicidality and seeks to provide empirical evidence for precise prevention and intervention for suicidal risks. The present study seeks to examine the relationship between mindsets and suicide ideation and the role of mindsets on the association between depression and suicide ideation. We hypothesized that 1) compared to non-fixed mindset, fixed mindset of depression, anxiety and stress 
predicts current and future suicidal ideation. 2) fixed mindset mediates the association between depression and suicidal ideation. We tested the hypotheses with a two-wave longitudinal study with baseline (Time 1) at the start of the academic school year (before the pandemic) and the Time 2 at the last month of the academic school year (during the pandemic) with a 9-month intervention. Hence, it is believed that changing the level of mindsets about mental health issues of young people with suicidality may be an effective way for suicide prevention.

\section{METHODS}

\section{Procedure and participants}

This was a two-wave longitudinal study among middle school students from eleven schools in Hong Kong. Invitations for study participation were sent to secondary schools in three main areas in Hong Kong, i.e., Hong Kong Island, Kowloon, and New Territory. Invitation would stop when at least one school in each of three of mediumof-instructions (English, Chinese, and both) joined the study in each of the three areas.

Parental consent and student assent were collected prior to the survey. Data were collected in classrooms by trained research assistants. The research assistants introduced the surveys to the students, ensured the students knew about their rights about voluntary participation, answered queries, and provided guidance on how to complete the questionnaire when appropriate. Students were reminded that their teachers were not able to access their responses to reduce the concern of being labelled and identified. After the students finished the surveys, research assistants packed and sealed the questionnaires and took the packages back to the office of the first author's university. Both English and Chinese versions of the questionnaire were prepared for participants' selection. Ethical approval was granted by the Human Subjects Ethics Sub-Committee of the first author's university (No. HSEARS20161222006). All participants in this study received souvenirs (worth US\$5) after finishing the follow-up questionnaire survey.

\section{Measures}

Suicidal ideation (SI) was assessed using the single-item suicidal ideation measure of the patient health questionnaire (PHQ-9, Kroenke et al., 2001), which was found to be a valid measure to screen suicide risk (Simon et al., 2013; Zhong et al., 2015). Respondents were asked "Over the last two weeks, how often have you been bothered by thoughts that you would be better off dead, or of hurting yourself in some way?" with answers ranging from 0 to $3(0=$ not at all, $1=$ several days, $2=$ more than half days, 3 = nearly every day). Answers of 1 to 3 were coded "yes" as having SI, while 0 was coded as "no" SI in past two weeks.

Fixed Mindsets was measured using the twelve-item Mindsets of Depression, Anxiety, and Stress Scale (MDASS, Zhu et al., 2021) to assess mindsets on depression, anxiety, and stress. An example item was "When you have a certain level of depression, you really cannot do much to change it". Each item was measured with a six-point Likert 
scale ( 1 to 3 = strongly disagree to slightly disagree; and 4-6 = slightly agree to strongly agree). Score 1-3 was recorded as 0, indicating a non-fixed mindset while scores 4-6 were recoded as 1, indicating fixed mindset. The sum score, ranged from 0 to 12 , reflects the level of fixed mindset. Both medians of T1 and T2 were 4 . We further dummy-coded the sum score into three groups: non-fixed mindset group (sum score $=$ zero), mild fixed mindset (sum score $=1$ to 3 ); strong fixed mindset (sum score $=4$ to 12). The Cronbach's $\alpha$ was 0.87 at $\mathrm{T} 1$ and 0.89 at $\mathrm{T} 2$.

Depressive symptoms were assessed using the eight-item patient health questionnaire (PHQ-8, Kroenke et al., 2009). Respondents were asked about the frequency of depressive symptoms in the past two weeks. An example item is "Little interest or pleasure in doing things" with answers ranging from 0 (not at all) to 3 (nearly every day). The sum of the scale (scores 0-24) was the indicator of depression, with higher scores indicating more depression. The Cronbach's $\alpha$ was 0.85 at T1 and 0.85 at T2.

Anxiety symptoms were measured using the seven-item generalized anxiety disorder scale (GAD-7, Spitzer et al., 2006). Respondents were asked how often the anxiety symptoms bothered them in the past two weeks. An example item was "How often did you feel nervous, anxious, or on edge in the past two weeks?" with a four-point Likert scale 0 to $3(0=$ not at all, $1=$ several days, $2=$ more than half days, $3=$ nearly every day). The sum of the scale (scores $0-21$ ) was the indicator of anxiety, with higher scores indicating more anxiety. The Cronbach's $\alpha$ was 0.92 at T1 and 0.93 at T2.

Covariate factors consist of socio-demographic data (age, gender) and socio-economic factors. Socioeconomic status (SES) was measured using the Family Affluence ScaleRevised (FAS-R) adapted from the FAS III (Hartley et. al., 2016). The FAS is one of the standardized measurements to measure SES for child or adolescent responders. The new and refined items included in adaptations were reflecting contemporary economic circumstances, technological advances, as well as cultural, social, and geographical norms in consumption (Currie et. al., 2008; Hartley et. al., 2016). The FAS-R consists of five components: house size (scores $0-3$ ), car (scores $0-1$ ), household appliances (scores 0 -2), learning devices (scores $0-2$ ), and internet accessibility (scores 0 -2), a total score ranging from 0 to 10 . The composite FAS-R score identified the responders into three levels of SES: low, medium, and high (Svedberg et. al., 2016).

\section{Data analyses}

A four-step process was adopted for data analyses. First, attribution analysis was used to test the potential influence of missing data. Differences in study variables were examined between participants who participated at $\mathrm{T} 1$ only and both $\mathrm{T} 1$ and $\mathrm{T} 2$. The two groups did not differ on any variables (all $p>.05$ ), suggesting that the data were missing at random. Second, descriptive analyses were used to compute the mean, standard deviation, skewness and kurtosis. Paired t tests were used to compare the T1 and T2 responses in depression, anxiety, mindset, and suicidal ideation. Spearman correlation was used to examine the correlation between variables. 
Third, the cross-sectional and longitudinal association between mindset of depression, anxiety, and suicidal ideation were examined using two hierarchical logistic regressions in three steps. In the first logistic regression, we examined the baseline factors and suicidal ideation after nine months (T2). T1 MDASS were entered in Step 1 to examine the association of baseline MDASS (T1) on T2 SI. MDASS were dummy-coded such that non-fixed mindset served as a reference group in comparison with mild and strong fixed mindset groups; Step 2 examined the association of T1 MDASS and T1 mental health symptoms with T2 SI; Step 3 examined the above associations after controlling covariates of gender, age, SES, T1 SI. Male, low SES group and non-suicidal ideation in $\mathrm{T} 1$ were reference groups. In the second logistic regression, we examined the association between T2 predictors and T2 SI. Step 1 examined the association between T2 MDASS and T2 SI; Step 2 examined the association among T2 MDASS, T2 depression and anxiety, and T2 SI; Step 3 examined the association after controlling covariates.

Fourth, two mediation hypotheses were tested using PROCESS models (Hayes, 2013). Following Hayes's suggestions, bootstrap analysis was conducted with 5000 iterations yielding 95\% confidence intervals and indirect effects. First, the mediation model of baseline mindset as mediator for the association between T1 depression and T2 SI was tested: T1 depression was entered as the predictor, T1 MDASS as the mediator, and T2 SI as the outcome. Second, we tested the mediation model of T2 mindset as mediator for the concurrent association between T2 depression and T2 SI. Gender, age, SES and $\mathrm{T} 1 \mathrm{SI}$ were included in all analyses as covariates.

\section{RESULTS}

\section{Attrition analysis}

At the baseline assessment (Time 1, T1), 1,491 students (695 male) from Grades 7 and 8 participated. Participants were aged between 10 to 16 years old with a mean age of $13.01(S D=0.86)$. The T1 measurements were carried out in September 2019 at the start of a new academic year. At Time 2 (T2) assessment in June 2020, at the end of the academic year, 1,393 students (640 males; $93.43 \%$ of the T1 sample) were retained and had a mean age of 13.04 ( $S D=0.85)$. In total, 1,393 students participated in both T1 and T2. Only participants validly completing both questionnaire assessments were entered into analyses.

Attrition analysis on depressive symptoms, anxiety symptoms, MDASS, and suicidal ideation were conducted between participants who provided complete data in two timepoints and those who provided data only at T1. Independent sample t-tests showed no statistically significant differences in depressive symptom $\left(t_{(1456)}=-1.37, p=.171\right)$, anxiety symptom $\left(t_{(1457)}=-0.96, p=.339\right)$, MDASS $\left(t_{(1413)}=-0.47, p=.642\right)$, and suicidal ideation (Chi-square $=.27, p=.626$ ) respectively between the participants in only $\mathrm{T} 1$ and those participated in $\mathrm{T} 1$ and $\mathrm{T} 2$.

\section{Descriptive statistics and correlation analyses}


Descriptive statistics for each measured variable and correlation analyses are summarized in Table 1. Paired sample t-tests were conducted to compare the changes in mindsets, depression, anxiety, and SI of T1 and T2 among the participants who provided complete data in two timepoints. Results showed participants reported lower depressive symptoms $\left(t_{(1380)}=2.86, p=.004\right)$, anxiety symptoms $\left(t_{(1350)}=5.60, p<.001\right)$, and suicidal ideation $\left(t_{(1380)}=2.44, p=.015\right)$, but higher fixed mindsets $\left(t_{(1222)}=-2.32\right.$, $p=.020)$ in $\mathrm{T} 2$ comparing to their responses in $\mathrm{T} 1$.

Correlational analyses indicated mental health measures and mindsets were significantly and positively associated with SI at both timepoints. Only gender among the covariate variables was significantly associated with depressive and anxiety symptom, mindsets, and SI at T2 (see Table 1). At Time 2, girls reported significantly more mental health symptoms, more fixed mindsets, and SI than boys.

\section{Relation between mindset and SI}

As for the hierarchical logistic regression on longitudinal association, results showed that adolescents with stronger fixed mindset at baseline were significantly more likely to have SI (crude model) after controlling for depression, anxiety, and demographic and SES variables (model 1$)$, and baseline SI (model $2\left(\mathrm{OR}_{\text {crude }}=4.38,95 \%\right.$ CI 2.42-7.92; $\mathrm{OR}_{\text {model } 1}=2.41 ; 95 \%$ CI 1.30-4.49; and $\mathrm{OR}_{\text {model } 2}=2.31 ; 95 \%$ CI 1.24-4.33). Mindsets of depression, anxiety, and stress in the crude model explained $7 \%$ of the variances in the second wave of SI in 9 months. Baseline depression and anxiety symptoms were significantly associated with SI but became insignificant after controlling for gender and baseline SI (see Table 2).

The cross-sectional association between fixed mindset and SI at T1 was also significant. Current strong fixed mindset was significantly and positively associated with SI in crude model and after controlling mental health, demographic and baseline SI (OR $\mathrm{OR}_{\text {crude }}$ $=12.60,95 \%$ CI 5.09-31.19; $\mathrm{OR}_{\text {Model } 1}=4.04 ; 95 \%$ CI 1.59-10.28; and $\mathrm{OR}_{\text {Model } 2}=3.47$; 95\% CI 1.35-8.93). Mild fixed mindsets were also significantly associated with SI $\left(\mathrm{OR}_{\text {crude }}=3.99 ; 95 \% \mathrm{CI} 1.55-10.28\right)$ after controlling mental health symptoms $(\mathrm{OR}=$ 2.71; 95\% CI 1.03-7.12). Mindsets of depression, anxiety, and stress in Model 1 explained $12 \%$ of the variances in the concurrent SI. The association of concurrent fixed mindset and SI was larger than that of both baseline and later SI. Both concurrent depression and anxiety were associated with SI, but their association coefficients were smaller than of the fixed mindsets and SI (see Table 3).

\section{Mediation role of fixed mindset on the association between depression and SI}

Two mediation models were conducted. The longitudinal mediation of T1 mindsets on the association between $\mathrm{T} 1$ depression on $\mathrm{T} 2$ SI (Mediation model: $\mathrm{T} 1 \mathrm{D} \rightarrow \mathrm{T} 1 \mathrm{MDASS} \rightarrow \mathrm{T} 2 \mathrm{SI}$ ), controlling T1 SI, demographic and SES factors (see Table 4). The effects of $\mathrm{T} 1$ depressive symptom on the mindsets $(b=.36,95 \%$ Boot $C I$ $=.32, .40)$, and T2 mindset on T2 SI $(b=.09,95 \%$ Boot $C I=.04, .13)$ were both positive and significant. The direct effect of T2 depression on T2 SI was significant 
( $b=.05,95 \%$ Boot $C I=.02, .09)$. The indirect effect (IE $=.03$ ) was statistically significant, 95\% Boot $C I=.014,05)$, indicating a positive predictive relationship between depression and SI as mediated through prior fixed mindsets of depression, anxiety and stress.

The cross-sectional model tested the mediation of $\mathrm{T} 2$ fixed mindsets on the association between T2 depression on T2 SI (Mediation model: T2D $\rightarrow$ T2MDAS $\rightarrow$ T2SI) controlling demographic and SES factors (see Table 5). The effects of T2 depressive symptom on the mindsets $(b=.30,95 \%$ Boot $C I=.26,34)$, and T2 mindset on T2 SI $(b=.17,95 \%$ Boot $C I=.12, .22)$ were both positive and significant. The direct effect of T2 depression on T2 SI was significant $(b=.21 .95 \%$ Boot $C I=.17, .25)$. In other words, the indirect effect $(\mathrm{IE}=.051)$ is statistically significant $(95 \%$ Boot $C I=.035$, 069), indicating a positive predictive relationship between depressive symptoms and SI as mediated through fixed mindsets of depression, anxiety and stress.

\section{DISCUSSION}

This study examines the association between mindsets and suicidality using a two-wave longitudinal study. It is found that fixed mindsets of depression, anxiety, and stress is a robust predictor of present and future SI. The mediation models showed that the association between depressive symptom and SI are partially mediated by fixed mindsets. This suggests that although depressive symptoms may increase the risk of SI and being fixated that the negative reactions to psychological strains cannot subside influences the way adolescents see their ways out to their temporary problems and thinking that death might be the only relief.

The findings about the strong association between mindsets and suicidality found in this study can be used to support the earlier postulations about the role and function of psychological pain in the development of suicidality proposed by (Shneidman, 1998) Suicide is an effort to stop the unbearable flow of negative affects (Shneidman, 1998) and when one suffers from depression, anxiety, and stress and thinks the symptoms are chronic and unmalleable, that person would be more likely to be despairing and frustrated than those who think the symptoms can be changed and improved later. Hopelessness also leads to suicidality (Van Orden et al., 2010); for instance, people with incurable diseases, such as AIDs or brain cancer, have higher risk for suicide because they are hopeless about recovery and endure pain and suffering of the disease (Van Orden et al., 2010). As depression, anxiety and stress cause psychological pain, fixed mindset of these symptoms may cause the suicidal fantasies and suicidal acts which are efforts to escape or put a stop to the psychological pain that is believed to be unchangeable (Shneidman, 1998). In fact, there is a daily saying in Chinese that "ai mo da yu xin si (哀莫大於心死)” which means that there is no sadness among all worse than being in despair) and this statement indicates the detrimental power of being hopeless and disbelieving in change. On the contrary, believing that psychological pain can be improved may ease the worry of enduring pain and may increase one's tolerance of current affective symptoms as bearable and temporary, which can foster hope during the difficult time. One who does not have hope any more is more likely to have an intention to die. 
It is also worth to mention that mindsets for psychological issues matter for suicidality because of its impact on attribution and emotion-regulation, which are strongly related to coping, help-seeking, and treatment choices (Kneeland, Dovidio, et al., 2016; Kneeland, Holen-Hoeksema, et al., 2016; Schroder, Kneeland, et al., 2019; Yeager et al., 2016). Similar to previous studies, the current study suggests people with affective symptoms had a 10-20\% higher chance of suicidality. Further, the current study found that having a strong fixed mindsets of depression, anxiety, and stress were associated with two to four times higher risk of endorsing SI. Negative emotions and psychological distresses may be inevitable. But the mindset on these ups and downs matters more. Fixed mindsets are associated with more negative attribution, less favorable emotion regulation, poor self-regulation and more hostile coping (Burnette et al., 2013; De Castella et al., 2013; Yeager et al., 2013; Yeager et al., 2011). On the contrary, growth mindsets buffer the influence of stress events and lead to more cognitive reappraisal, active help-seeking and choosing effortful treatment, and better adjustment to life changes (Schroder, Kneeland, et al., 2019; Schroder et al., 2017; Zhu, Ni, et al., 2020). The mindset on psychological distress and subsequent coping behaviors, other than simply the distress itself, may matter more on whether the suicidal thoughts are triggered.

The findings of current research have strong potential clinical implications. First, as mindsets of depression, anxiety and stress can play a role in emotion regulation,, intervention aimed at decreasing fixed mindset and strengthening growth mindset is highly likely to increase perceived control of the symptoms and positive coping. Also, literature supports the efficacy of brief growth mindset intervention (Schleider \& Weisz, 2018).Such intervention on promoting growth mindset on depression, anxiety and stress would be highly potential and feasible in decreasing the risk of suicidal thoughts. Second, the current study found fixed mindsets of depression, anxiety, and stress are a robust predictor of current and future SI. As early identification and assessment are important to prevent suicide (WHO, 2021), the scale of fixed mindset of depression, anxiety, and stress may be useful as a complementary measure for screening of suicidality. Third, the findings of the current study have the potential to develop scalable school intervention to promote positive attitude and coping with mental health symptoms. Also fostering growth mindset about mental health symptoms would be helpful to decrease the psychological burden for adolescents in seeking help, thereby decreasing the negative impact of stigma of mental health problems.

\section{Limitations}

The current research had several limitations. First, the measures were self-reported and were subject to the influence of social desirability despite the fact that the study was already administered by trained research assistants in the classrooms and participants were reassured that their answers were confidential. Second, we used a single-item dichotomous measure of SI and were unable to examine the severity of suicidality. Considering the limited survey time in classroom in half-day school resumption during 
COVID-19 pandemic and the concerns about asking extensively about suicidality during the COVID-19 period, a validated single-item measure of SI was the most feasible approach to measure SI. Third, this study examines the association of mindsets and suicidality among a community sample of adolescents. Further studies should also examine the association among clinical samples with depression and different age cohorts to expand the generalizability.

Despite the aforementioned limitations, this study provides empirical support to the classic theoretical postulation that psychological pain, tunnel vision, and suicidality are intertwined using longitudinal data and that provides a very strong argument about the nature of causal relationships between mindset and suicidal ideation (Jose, 2016) which deserves more examination. Also, this study marks an important step forward in understanding how mindsets or implicit theories are associated SI. Our specific focus on the mindsets on depression, anxiety, and stress, instead of the mindset of intelligence and personality, is a strength of our study. The focus on the interaction between mindsets of mental health as a mechanism underlying the relation of depression and suicidality addresses a specific avenue for suicide prevention among people who suffer from depression.

\section{CONCLUSION}

The present study is the first to provide empirical evidence for the association among depression symptoms and mindsets of psychological issues, and SI and to examine the mediation association of mindset on the relationship between depressive symptoms and SI. The results of the study suggest that the mindsets of depression, anxiety and stress scale (MDASS) is a robust and strong tool for screening current and future suicidal risk. The findings also invite the formulation of interventions aim at reducing the fixed mindsets of depression, anxiety and stress or promoting malleability belief of psychological distresses, which may reduce the risks of adolescent suicidality. 


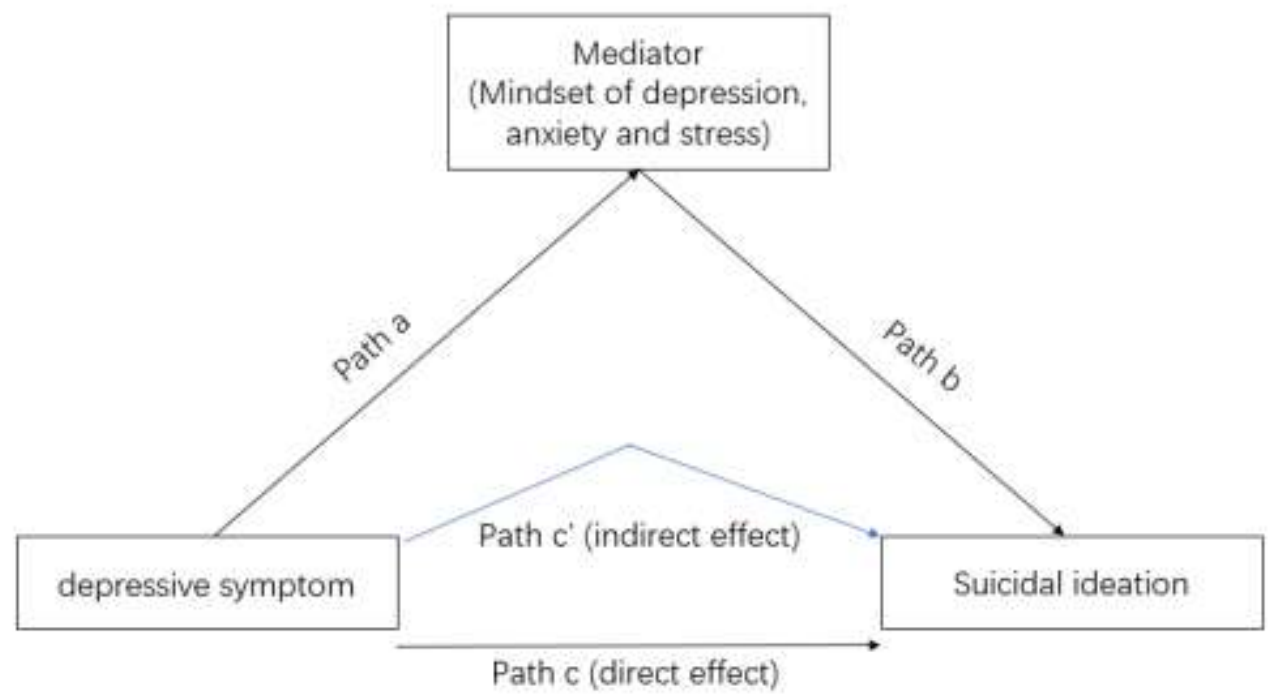

Figure 1. Sample mediation model. 
Table 1. Descriptive Statistics and Correlation Analysis $(\mathrm{N}=1,393)$

\begin{tabular}{lcccccccc}
\hline & 1 & 2 & 3 & 4 & 5 & 6 & 7 & 8 \\
\hline 1. T1 Depression & - & & & & & & & \\
2. T1 Anxiety & .80 & - & & & & & & \\
3. T1 MDASS & .51 & .52 & - & & & & & \\
4. T1 Suicidal Ideation & .53 & .53 & .35 & - & & & & \\
5. T2 Depression & .50 & .47 & .33 & .27 & - & & & \\
6. T2 Anxiety & .42 & .47 & .31 & .26 & .79 & - & & \\
7. T2 MDASS & .33 & .34 & .43 & .21 & .44 & .44 & - & \\
8. T2 Suicidal Ideation & .28 & .29 & .24 & .30 & .49 & .53 & .37 & - \\
$\quad$ Skewness & 1.22 & 1.22 & 0.33 & 1.19 & 1.23 & 1.59 & 0.33 & 1.42 \\
$\quad$ Kurtosis & 1.80 & 1.03 & -1.00 & -0.59 & 1.47 & 2.16 & -0.96 & 0.02 \\
$\quad$ Mean or \% & 5.27 & 4.69 & 4.81 & $24.46 \%$ & 5.13 & 3.93 & 5.06 & $21.04 \%$ \\
$\quad$ SD & 4.58 & 4.83 & 3.45 & - & 4.70 & 4.87 & 3.56 & - \\
\hline
\end{tabular}

All correlation coefficients are significant at the 0.001 level (2-tailed).

$\mathrm{T} 1=$ Time $1 ; \mathrm{T} 2=$ Time $2 ;$ MDASS $=$ mindset of depression, anxiety, and stress scale Depression: PHQ-8 score ranges from 0-24.

Anxiety: GAD-7 score ranges from 0-21.

Suicidal Ideation: the $9^{\text {th }}$ item of PHQ-9 score range from 0-1.

MDASS: fixed mindset of depression, anxiety and stress score range from 0-12. 
Table 2. Hierarchical Logistic Regression Coefficients and 95\% confidence intervals for the relationship between Time 1 Mindset and Time 2 Suicidal Ideation

\begin{tabular}{lllllll}
\hline & \multicolumn{2}{l}{ Crude model } & \multicolumn{2}{l}{ Model 1 } & \multicolumn{3}{l}{ Model 2 } \\
\cline { 2 - 7 } Predictors & OR & $95 \%$ CI & OR & $95 \%$ CI & OR & $95 \%$ CI \\
\hline T1 Mindset (non-fixed) & ref & & ref & & ref & \\
T1 Mindset (mild fixed) & 1.75 & $0.93-3.30$ & 1.52 & $0.80-2.88$ & 1.51 & $0.79-2.88$ \\
T1 Mindset (strong fixed) & $4.38^{* *}$ & $2.42-7.92$ & $2.41^{* *}$ & $1.30-4.49$ & $\mathbf{2 . 3 1}$ & $1.24-4.33$ \\
T1 Depression & & & $1.63^{*}$ & $1.09-2.43$ & 1.43 & $0.94-2.16$ \\
T1 Anxiety & & & $1.06^{*}$ & $1.01-1.11$ & 1.03 & $0.99-1.08$ \\
Gender (female) & & & & & $\mathbf{1 . 5 7}^{* *}$ & $1.15-2.13$ \\
Age & & & & & 0.87 & $0.74-1.04$ \\
SES group & & & & & ref & \\
SES group (1) & & & & & 0.98 & $0.63-1.55$ \\
SES group (2) & & & & & 1.16 & $0.70-1.93$ \\
T1 Suicidal Ideation (Y) & & & .128 & & $\mathbf{2 . 5 8 ^ { * * * }}$ & $1.80-3.69$ \\
Pseudo $R^{2}$ & .067 & & & & & \\
\hline
\end{tabular}

T1 $=$ Time $1 ;$ SES $=$ socio-economic status.

OR $=$ Odds Ratio $; 95 \% \mathrm{CI}=95 \%$ Confidence Interval; Pseudo- $R^{2}=$ Nagelkerke's $\mathrm{R}^{2}$

${ }^{*} p<.05 ;{ }^{* *} p<.01 ;{ }^{* * *} p<.001$.

Table 3. Hierarchical Logistic Regression Coefficients and 95\% confidence intervals for the relationship between Time 2 Mindset and Time 2 Suicidal Ideation

\begin{tabular}{lllllll}
\hline & \multicolumn{2}{l}{ Crude model } & \multicolumn{2}{l}{ Model 1 } & \multicolumn{3}{l}{ Model 2 } \\
\cline { 2 - 7 } Predictors & OR & $95 \%$ CI & OR & $95 \%$ CI & OR & 95\% CI \\
\hline T2 Mindset (non-fixed) & ref & & ref & & ref & \\
T2 Mindset (mild fixed) & $3.99^{* *}$ & $1.55-10.28$ & $2.71^{*}$ & $1.03-7.12$ & 2.42 & $0.91-6.42$ \\
T2 Mindset (strong fixed) & $12.60^{* * *}$ & $5.09-31.19$ & $4.04^{* *}$ & $1.59-10.28$ & $3.47^{* *}$ & $1.35-8.93$ \\
T2 Depression & & & $2.62^{* * *}$ & $1.70-4.04$ & $2.33^{* * *}$ & $1.49-3.63$ \\
T2 Anxiety & & & $1.18^{* * *}$ & $1.12-1.24$ & $1.18^{* * *}$ & $1.12-1.24$ \\
Gender (female) & & & & 1.12 & $0.78-1.61$ \\
Age & & & & 0.86 & $0.71-1.06$ \\
SES group & & & & ref & \\
SES group (1) & & & & 0.98 & $0.57-1.69$ \\
SES group (2) & & & & 1.38 & $0.76-2.52$ \\
T1 Suicidal Ideation (Y) & & & & & $2.70^{* * *}$ & $1.87-3.89$ \\
Pseudo- $R^{2}$ & .116 & & .385 & & .416 & \\
\hline
\end{tabular}

$\mathrm{T} 1=$ Time $1 ; \mathrm{T} 2=$ Time $2 ; \mathrm{SES}=$ socio-economic status.

OR $=$ Odds Ratio $; 95 \% \mathrm{CI}=95 \%$ Confidence Interval. Pseudo- $R^{2}=$ Nagelkerke's $\mathrm{R}^{2}$

${ }^{*} p<.05 ;{ }^{* *} p<.01 ;{ }^{* * * *} p<.001$. 
Table 4. Regression Results of Testing the Mediation of T1 Mindset in the Relationship between T1 Depression and T2 Suicidal Ideation

\begin{tabular}{lcccccc}
\hline & $B$ & SE & $t / z$ & $p$ & LLCI & ULCI \\
\hline $\begin{array}{l}\text { Effect of T1 depression } \\
\text { on T1 mindset (a) }\end{array}$ & .359 & .022 & 16.442 & .000 & .316 & .402 \\
$\begin{array}{l}\text { Effect of T1 mindset on } \\
\text { T2 SI (b) }\end{array}$ & .087 & .025 & 3.535 & .000 & .039 & .135 \\
$\begin{array}{l}\text { Direct effect of T1 } \\
\text { depression on T2 SI (c) }\end{array}$ & .054 & .019 & 2.787 & .005 & .016 & .092 \\
$\begin{array}{l}\text { Indirect effect (c') } \\
\text { bootstrap }\end{array}$ & .031 & .009 & & & .014 & .050 \\
$\begin{array}{l}\text { T1 = Time 1; T2 = Time 2; SI = Suicidal Ideation; LLCI and ULCI are the 95\% bootstrap confidence } \\
\text { intervals from 5000 bootstrap replicates. }\end{array}$
\end{tabular}

Table 5. Regression Results of Testing the Mediation of T2 Mindset in the Relationship between T2 Depression and T2 Suicidal Ideation

\begin{tabular}{lcccccc}
\hline & $B$ & SE & $t / z$ & $p$ & LLCI & ULCI \\
\hline $\begin{array}{l}\text { Effect of T2 depression } \\
\text { on T2 mindset }(a)\end{array}$ & .303 & .021 & 14.496 & .000 & .262 & .344 \\
$\begin{array}{l}\text { Effect of T2 mindset on } \\
\text { T2 SI }(b)\end{array}$ & .169 & .027 & 6.302 & .000 & .117 & .222 \\
$\begin{array}{l}\text { Direct effect of T2 } \\
\text { depression on T2 SI }(c)\end{array}$ & .210 & .021 & 10.084 & .000 & .170 & .251 \\
$\begin{array}{l}\text { Indirect effect (c') } \\
\text { bootstrap }\end{array}$ & .051 & .01 & & & .034 & .069 \\
$\begin{array}{l}\text { T1 = Time 1; T2 = Time 2; SI = Suicidal Ideation; LLCI and ULCI are the 95\% bootstrap confidence } \\
\text { intervals from 5000 bootstrap replicates. }\end{array}$
\end{tabular}




\section{References}

Burnette, J. L., O'Boyle, E. H., VanEpps, E. M., Pollack, J. M., \& Finkel, E. J. (2013). Mind-sets matter: a meta-analytic review of implicit theories and selfregulation. Psychol Bull, 139(3), 655-701. https://doi.org/10.1037/a0029531

Chu, C., Buchman-Schmitt, J. M., Stanley, I. H., Hom, M. A., Tucker, R. P., Hagan, C. R., Rogers, M. L., Podlogar, M. C., Chiurliza, B., Ringer, F. B., Michaels, M. S., Patros, C. H. G., \& Joiner, T. E., Jr. (2017). The interpersonal theory of suicide: A systematic review and meta-analysis of a decade of cross-national research [Article]. Psychological Bulletin, 143(12), 1313-1345. https://doi.org/10.1037/bul0000123

De Castella, K., Goldin, P., Jazaieri, H., Ziv, M., Dweck, C. S., \& Gross, J. J. (2013). Beliefs about emotion: Links to emotion regulation, well-being, and psychological distress. Basic and Applied Social Psychology, 35(6), 497-505. https://doi.org/10.1080/01973533.2013.840632

Dweck, C. S. (2013). Self-theories: Their role in motivation, personality, and development. Psychology Press.

Dykxhoorn, J., Hatcher, S., Roy-Gagnon, M.-H., \& Colman, I. (2017). Early life predictors of adolescent suicidal thoughts and adverse outcomes in two population-based cohort studies. PLoS ONE, 12(8), e0183182.

Evans, E., Hawton, K., \& Rodham, K. (2004). Factors associated with suicidal phenomena in adolescents: a systematic review of population-based studies. Clinical psychology review, 24(8), 957-979.

Jose, P. E. (2016). The merits of using longitudinal mediation. Educational Psychologist, 51(3-4), 331-341.

King, R. B., \& dela Rosa, E. D. (2019). Are your emotions under your control or not? Implicit theories of emotion predict well-being via cognitive reappraisal [Article]. Personality and Individual Differences, 138, 177-182. https://doi.org/10.1016/j.paid.2018.09.040

Kneeland, E. T., Dovidio, J. F., Joormann, J., \& Clark, M. S. (2016). Emotion malleability beliefs, emotion regulation, and psychopathology: Integrating affective and clinical science [Review]. Clinical Psychology Review, 45, 8188. https://doi.org/10.1016/j.cpr.2016.03.008

Kneeland, E. T., Holen-Hoeksema, S., Dovidio, J. F., \& Gruber, J. (2016). Emotion malleability beliefs influence the spontaneous regulation of social anxiety. Cognitive Therapy and Research, 40(4), 496-509. https://doi.org/10.1007/s10608-016-9765-1

Mullarkey, M. C., \& Schleider, J. L. (2020). Contributions of fixed mindsets and hopelessness to anxiety and depressive symptoms: A commonality analysis approach [Article]. Journal of Affective Disorders, 261, 245-252. https://doi.org/10.1016/i.jad.2019.10.023

Olié, E., Guillaume, S., Jaussent, I., Courtet, P., \& Jollant, F. (2010). Higher psychological pain during a major depressive episode may be a factor of vulnerability to suicidal ideation and act. Journal of Affective Disorders, 120(1-3), 226-230.

Schleider, J. L., Abel, M. R., \& Weisz, J. R. (2015). Implicit theories and youth mental health problems: A random-effects meta-analysis. Clinical Psychology Review, 35, 1-9. https://doi.org/10.1016/j.cpr.2014.11.001

Schleider, J. L., Abel, M. R., \& Weisz, J. R. (2019). Do immediate gains predict longterm symptom change? Findings from a randomized trial of a single-session intervention for youth anxiety and depression. Child Psychiatry and Human 
Development, 50(5), 868-881. https://doi.org/10.1007/s10578-019-00889-2

Schleider, J. L., Burnette, J. L., Widman, L., Hoyt, C., \& Prinstein, M. J. (2019).

Randomized trial of a single-session growth mind-set intervention for rural adolescents' internalizing and externalizing problems. Journal of Clinical Child and Adolescent Psychology. https://doi.org/10.1080/15374416.2019.1622123

Schleider, J. L., \& Schroder, H. S. (2018). Implicit theories of personality across development: Impacts on coping, resilience and mental health. In V. ZeiglerHill \& T. K. Shackelford (Eds.), The SAGE handbook of personality and individual differences: The science of personality and individual differences (pp. 152-170, Chapter xxiv, 646 Pages). Sage Reference. https://doi.org/10.4135/9781526451163.n7

Schleider, J. L., \& Weisz, J. (2018). A single-session growth mindset intervention for adolescent anxiety and depression: 9-month outcomes of a randomized trial [Article]. Journal of Child Psychology and Psychiatry and Allied Disciplines, 59(2), 160-170. https://doi.org/10.1111/jcpp.12811

Schroder, H. S. (2018). Malleability beliefs of anxiety: Impact on treatment preferences and emotion regulation. Michigan State University. Psychology.

Schroder, H. S. (2020). Mindsets in the clinic: Applying mindset theory to clinical psychology. Clinical Psychology Review, 101957.

Schroder, H. S., Callahan, C. P., Gornik, A. E., \& Moser, J. S. (2019). The fixed mindset of anxiety predicts future distress: A longitudinal study. Behavior Therapy, 50(4), 710-717. https://doi.org/http://dx.doi.org/10.1016/j.beth.2018.11.001

Schroder, H. S., Dawood, S., Yalch, M. M., Donnellan, M. B., \& Moser, J. S. (2016). Evaluating the domain specificity of mental health-related mind-sets. Social Psychological and Personality Science, 7(6), 508-520. https://doi.org/10.1177/1948550616644657

Schroder, H. S., Kneeland, E. T., Silverman, A. L., Beard, C., \& Björgvinsson, T. (2019). Beliefs about the malleability of anxiety and general emotions and their relation to treatment outcomes in acute psychiatric treatment. Cognitive Therapy and Research, 43(2), 312-323. https://doi.org/10.1007/s10608-0189985-7

Schroder, H. S., Yalch, M. M., Dawood, S., Callahan, C. P., Brent Donnellan, M., \& Moser, J. S. (2017). Growth mindset of anxiety buffers the link between stressful life events and psychological distress and coping strategies [Article]. Personality and Individual Differences, 110, 23-26. https://doi.org/10.1016/j.paid.2017.01.016

Shneidman, E. S. (1998). Further reflections on suicide and psychache. Suicide \& lifethreatening behavior, 28(3), 245.

Tezanos, K. M., Pollak, O. H., \& Cha, C. B. (2021). Conceptualizing death: How do suicidal adolescents view the end of their lives? Suicide and Life Threatening Behavior.

Van Orden, K. A., Witte, T. K., Cukrowicz, K. C., Braithwaite, S. R., Selby, E. A., \& Joiner Jr, T. E. (2010). The Interpersonal Theory of Suicide [Article]. Psychological Review, 117(2), 575-600. https://doi.org/10.1037/a0018697

World Health Organization. (2014). Suicide Worldwide in 2019: GLobal Health Estimates. https://apps.who.int/iris/bitstream/handle/10665/131056/9789241564878_eng. pdf 
World Health Organization. (2021). Suicide worldwide in 2019: global health estimates (9240026649).

https://apps.who.int/iris/bitstream/handle/10665/341728/9789240026643eng.pdf?sequence $=1$

Yeager, D. S., Lee, H. Y., \& Jamieson, J. P. (2016). How to improve adolescent stress responses: Insights from integrating implicit theories of personality and biopsychosocial models. Psychological Science, 27(8), 1078-1091. https://doi.org/http://dx.doi.org/10.1177/0956797616649604

Yeager, D. S., Miu, A. S., Powers, J., \& Dweck, C. S. (2013). Implicit theories of personality and attributions of hostile intent: A meta-analysis, an experiment, and a longitudinal intervention. Child Development, 84(5), 1651-1667. https://doi.org/10.1111/cdev.12062

Yeager, D. S., Trzesniewski, K. H., Tirri, K., Nokelainen, P., \& Dweck, C. S. (2011). Adolescents' implicit theories predict desire for vengeance after peer conflicts: Correlational and experimental evidence. Developmental Psychology, 47(4), 1090-1107. https://doi.org/http://dx.doi.org/10.1037/a0023769

Zhu, S., Ni, S., \& Hamilton, K. (2020). Cognition malleability belief, emotion regulation and adolescent well-being: examining a mediation model among migrant youth. Health Psychology and Behavioral Medicine. https://doi.org/10.1080/21642850.2020.1806717

Zhu, S., Zhuang, Y., \& Cheung, S. H. (2020). Domain specificity or generality: Assessing the Chinese Implicit Theories Scale of six fundamental psychological attributes. Frontiers in Psychology. https://doi.org/10.3389/fpsyg.2020.00142

Zhu, S., Zhuang, Y., \& Lee, P. (2021). Psychometric properties of the Mindsets of Depression, Anxiety, and Stress Scale (MDASS) in Chinese young adults and adolescents Early Intervention in Psychiatry. https://doi.org/https://doi.org/10.1111/eip.13177

Zimmermann, M., Hmaidan, R., Preiser, B., \& Papa, A. (2020). The Influence of Implicit Theories of Depression on Treatment-Relevant Attitudes [Article]. Cognitive Therapy and Research, 44(5), 1002-1014. https://doi.org/10.1007/s10608-020-10120-5 